Siebzehn von den 25 Karten stellen die Verbreitung und Produktion der wichtigsten Güter der Erde dar. Davon gelten 10 dem landwirtschaftlichen Bereich. Beachtung finden: Holz - Baumwolle, Wolle, Rohseide - Kautschuk - verschiedene Getreide - Kartoffel - Rohr- und Rübenzucker, Kaffee, Kakao, Tee - Vegetabile Fette und Öle - Rinder, Schweine, Schafe - Fischerei. Die 7 andern Karten illustrieren die verfügbaren Wasserkräfte und die Kapazität der Elektrizitätsgewinnung, die Förderung und den Handel von Erdöl, Steinkohle und Eisenerz, die Gewinnung von weiteren 17 Montanprodukten, die Eisen- und Stahlproduktion, sowie die Rohstoffversorgung und Herstellung der Leichtmetalle Aluminium und Magnesium. Alle diese Darstellungen sind auswertbare Punktkarten und gestatten deshalb ein Auszählen der gewerteten Signaturen, die zugleich den Ort der Produktion belegen. Die übrigen Karten sind vornehmlich zum Vergleichen und Verknüpfen des analytisch behandelten Tatsachenmateriales bestimmt. Sie demonstrieren: Relief, Klima- resp. Vegetationstypen, Bevölkerungsverteilung, Welthandelswege, Industrialisierung, Wirtschafts- und Außenhandelsstruktur. Davon sind die ersten 4 als sog. Grundlagenkarten auf Pauspapier gedruckt. Als solche können sie jeder anderen Karte aufgelegt werden. Dadurch wird das Herausarbeiten von Beziehungen erleichtert.

Der beiliegende Text ist streng nach den Einzelkarten gegliedert. Er erläutert einmal die Legende, weist auf die statistischen und kartographischen Schwierigkeiten der Ausführung hin und berichtet von den Überlegungen, die zur gewählten Darstellung geführt haben. Sodann beschreibt er die wirtschaftliche Eigenart des dargestellten Produktes, gibt Aufschluß über Handel und Konsum und skizziert die seit den Vorkriegsjahren (1937/38) eingetretenen Veränderungen. Wertvoll sind ferner die Anregungen zur Auswertung und zum Vergleich der Einzelkarten.

Die aktuelle Dokumentation modernen Wirtschaftsgeschehens ist reichhaltig und vielseitig. Kaufleute und Volkswirtschafter schätzen ihren praktischen Wert. Doch bietet ihr Studium auch dem Laien großen Gewinn. Besonderen Anklang wird der „Wirtschaftsgeographische Atlas“ beim Lehrer finden. Ist er doch in vielen Einzelheiten auf die Bedürfnisse der Schule, des Unterrichtes in Geographie, $\mathrm{W}$ rirtschafts- und Warenkunde zugeschnitten. Seine Punktkarten ergänzen mit Vorteil die qualitativ nicht wertbaren Wirtschaftskarten der gebräuchlichen Schulatlanten. Die handlichen Einzelblätter können im Schaukasten ausgestellt oder mit dem Episkop projiziert werden. Im Sinne des auch auf der Mittelschulstufe zu pflegenden Arbeitsunterrichtes gehören die leicht faßlichen Karten auch in die Hand des Schülers. Darum ist die Bitte wohl nicht abwegig, es möge der Verlag an Schulen auch Einzelblätter abgeben.

\title{
DIE MODERNE KARTOGRAPHIE ZU EINEM BERICHT DER UNO
}

\author{
Franz Flury
}

\author{
(Modern cartography, Base Maps for World Needs, United Nations, Department of social affairs, \\ Lake Succeess - Nerw York)
}

Vor kurzem publizierte die UNO einen Bericht über den Stand der Kartographie der Erde und ihre wünschenswerte Zukunftsgestaltung, der wert ist, auch hier kommentiert zu werden. Die Schrift enthält zwei Teile, einen Expertenbericht und eine Abhandlung über moderne kartographische Methoden, welch letztere vom panamerikanischen Institut für Geographie und Geschichte stammt. Der Expertenbericht wurde verfaßt von den Herren R. L. Brown, Direktor des Ordnance Survey von Großbritannien, LeITE DE CASTRo, Generalsekretär des nationalen Rates für Geographie von Brasilien, H. Randall, Präsident der Kommission für Kartographie des panamerikanischen Instituts für Geographie und Geschichte, W. SchERmerhorn, gew. Präsident der internationalen Gesellschaft für Photogrammetrie und R. VerLaine, Chef des photogrammetrischen Dienstes des militärgeographischen Institutes Belgiens.

Im Expertenbericht wird vorerst der Begriff der Kartographie umschrieben, der hier vom ersten Beginn einer Aufnahme bis zum Vorliegen der fertigen Karte alles umfaßt. Unter voller Anerkennung des bisher in der kartographischen Aufnahme der Welt geleisteten, geben die Experten ihrer Überraschung Ausdruck über den Umfang dessen, was noch zu tun übrig bleibt. Für diesen Zustand machen sie in erster Linie die mangelnde Einsicht mancher Regierungen verantwortlich, den Mangel an technischem Personal und Material, sowie das Fehlen einer internationalen technischen Organisation an welche sich die Staaten wenden könnten. - Anderseits kann der einzige Berufskartograph im Generalsekretariat der Uno unmöglich den wachsenden Ansprüchen an kartographische Auskünfte gerecht werden. Die Experten bestehen darauf, daß gute Karten unentbehrlich sind. Sie begründen dies mit der Notwendigkeit der Ausführung der gewaltigen Projekte, welche die Ernährung der ständig wachsenden Menschheit sicherstellen sollen. Die Erfahrung hat jedoch gezeigt, daß solche Arbeiten nur rationell durchgeführt werden können, sofern genügende topographische Karten zur Verfügung stehen. Die Erstellung topographischer Karten ist aber eine 
Aufgabe, jahrhundertealte Erfahrung hat das gezeigt, die nur vom Staat einwandfrei gelöst werden kann. Dagegen braucht die Erstellung von Karten zu besonderen Zwecken nicht Sache des Staates allein zu sein.

Sollen gute topographische Karten erstellt werden, so ist das erste Erfordernis die Schaffung einer zuverlässigen geodätischen Grundlage. Ein ausgeglichenes Dreiecknetz, das sich über die ganze Erde erstreckt, mit ungefähr $50 \mathrm{~km}$ Seitenlänge z. B. existiert leider noch nicht. Dagegen bestehen zahlreiche, zuverlässige nationale Triangulationen. Die internationale Koordination derselben ist eine neue, dringende Aufgabe der Staaten.

Eine der wichtigsten Aufnahmemethoden für topographische Karten ist die Flugphotogrammetrie. Die Flugbilder bieten darüber hinaus einen weitgehenden Einblick in die geographischen Verhältnisse, ja, lassen bekanntlich bereits die Grundzüge der Geologie, sogar im urwaldbedeckten Gebiet erkennen. Ist vor der Erschließung einer Gegend eine topographische Aufnahme undurchführbar, so ist die Aufnahme des Geländes aus der Luft umso notwendiger und zwar unter der Aufsicht eines erfahrenen photogrammetrischen Technikers, damit nachträglich eine topographische Karte kleinen Maßstabs (Explorationsaufnahme) erstellt werden kann.

Der Stand der Kartographie in der Welt. Bloß für weniger als $2 \%$ der Erdoberfläche existieren Karten im Maßstab 1:25000 und größer und wahrscheinlich für nicht mehr als 25\% der Erdoberfläche existieren sog. Explorationskarten in Maßstäben um 1:250000 und 1:300000. Ausgenommen einige europäische Nationen und begrenzte andere Gebiete, ist der Stand der Kartographie nirgends genügend hoch, um eine vollständige wirtschaftliche Entwicklung der natürlichen Hilfsquellen zu ermöglichen.

Dieser bedauernswerte Zustand wird durch zwei Karten illustriert. Es muß jedoch ein wesentlicher Fehler hervorgehoben werden, indem die Schweiz in niedererem Rang dargestellt wird als die umliegenden europäischen Staaten. $\mathrm{Da}$ einer der Hauptgründe für die ungenügende vermessungstechnische Erschließung der Welt der Mangel an internationaler Zusammenarbeit ist, konnte den Experten nicht entgehen und um Abhilfe zu schaffen, schlagen sie eine dreistufige Organisation vor: r. Intergouvernementale, weltumspannende, auf einen Gegenstand spezialisierte Organisationen (Beispiel : Die internationale Organisation der Zivilluftfahrt), 2. Intergouvernementale, regionale Organisationen, welche sich mit dem ganzen Gebiet der Kartographie in weitem Sinne befassen (Beispiel : Das Panamerikanische Institut für Geographie und Geschichte), 3, Internationale wissenschaftliche nichtgonvernementale Organisationen (Beispiel: Die internationale Gesellschaft für Photogrammetrie). Ob nun die dringend notwendige internationale Zusammenarbeit im Vermessungswesen so oder anders organisiert wird, die Hauptsache ist die Realisation derselben und da muß leider an die Schwierigkeit, so viele Regierungen unter einen Hut zu bringen, erinnert werden.

Um dem enormen Bedarf der UNO und ihrer Kommissionen an zuverlässigem kartographischem Material zu entsprechen, machen die Experten großzügige Vorschläge: Abhaltung von Regionalversammlungen von kartographischen Technikern zur Förderung der kartographischen Aufnahme gewisser Regionen nach dem erfolgreichen Beispiel der Kartographischen Kommission des Panamerikanischen Instituts für Geographie und Geschichte. 2. Die Einrichtung eines Kartographischen Bureaus der Vereinigten Nationen: Es hat a) das Kartenmaterial zu beschaffen für alle Organe der UNO, gegebenenfalls das benötigte Material zu erstellen, b) die kartographische Tätigkeit in der UNO zu koordinieren und zu zentralisieren, c) gegebenenfalls die Reproduktionserlaubnis einzuholen, Aufstellung einer für die Dokumente der UNO verbindlichen geographischen Nomenklatur in Zusammenarbeit mit den nationalen Nomenklaturkommissionen, Vorbereitung der Regionalversammlungen, Herausgabe eines Jahrbuchs der Weltkartographie mit Spezialbibliographie über neue kartographische Methoden zu besorgen. Zur Erledigung dieses vollgerüttelten Maßes von Aufgaben ist ein entsprechender Stab von Fachleuten vorgesehen, sowie eine Reihe von fachmännischen Beratern. Die Tätigkeit dieses kartographischen Bureaus soll in jährlichen Versammlungen überprüft werden, die aus Vertretetn der Regionalversammlungen, der interessierten Institutionen der UNO und der internationalen technischen und wissenschaftlichen Fachverbände zusammengesetzt sein sollen. Mit Beschluß vom 27. Juli 1949 beauftragte der Conseil économique et social des Nations Unies den Generalsekretär der UNO, die Empfehlungen der Experten ins Werk zu setzen.

Auf die grundlegende Bedeutung dieses Eingreifens der UNO in die kartographische Erforschung der Welt sei besonders hingewiesen. Das Ergebnis wird selbstverständlich nicht nur von der ausgeklügelten Organisation, sondern auch von den leitenden Persönlichkeiten abhängen. Immerhin steht zu hoffen, da $\beta$ auch von dieser Seite ein Beitrag zu der dringend notwendigen besseren kartographischen Erforschung der Erde geleistet wird.

Bericht über moderne kartographische Methoden, verfaßt von der kartographischen Kommission des panamerikanischen Instituts fiir Geographie und Geschichte. Diese kartographische Kommission bezweckt, die Kartographie in den Staaten der westlichen Halbkugel gemäß ihrem Bedarf an Karten zu fördern, ihren Ausbau zu erleichtern und zu beschleunigen. Die elektronischen Methoden dienten zur Sicherung der Navigation der Schiffe und Flugzeuge im Kriege. Es hat sich gezeigt, daß solche Methoden für Vermessungszwecke, zur Orts- und Distanzbestimmung verwendet werden können. Man hofft sogar durch in der Luft beförderte elektronische Apparate die großen Distanzen zwischen den Kontinenten mit geodätischer Genauigkeit bestimmen zu können. Der Bericht von 
C. A. HaRT über diese Methoden erschien in den Comptes-rendus der Sitzung der Union géodésique et géophysique internationale in Oslo 1948.

Anders als die topographischen und hydrographischen Karten, beruhen die Fliegerkarten auf bereits bestehenden Karten und nicht auf direkten Aufnahmen. Die Projektion muß sorgfältig mit Rücksicht auf möglichst geringe Verzerrung gewählt werden. Von den Spezialkarten verschiedener Maßstäbe heben wir nur die Fliegerkarte 1: 1000000 hervor, die von allen Fliegern benützt wird und welche die einzige Serie von Karten in diesem Maßstab ist, welche die ganze Erdoberfläche bedeckt (genannt WAC $=$ World Aeronautical Chart). Die Fliegerkarten genießen den Vorteil der Einheitlichkeit, den viele andere Karten nicht aufiveisen, indem die meisten wichtigen Länder die Vorschriften der OACI für die Erstellung dieser Karten angenommen haben (Annexe 4 à la Convention relative à l'Aviation civile internationale $\alpha$ Standards et pratiques recommandés : Cartes aéronautiques ". OACI, Montréal, Province de Québec, Canada).

Karten fïr spezielle Zrwecke : Es handelt sich um Karten, die irgend ein Phänomen, z. B. einen Aspekt der Nutzbarmachung der Erde oder der Beziehungen zwischen den Menschen darstellen. Hier wird nun eine originelle und ausführliche Anleitung über das zweckmäßigste Vorgehen bei der Erstellung solcher Karten, an der es bisher fehlte, gegeben. Es sei ausdrücklich darauf verwiesen; sie ist nicht nur für die Kartographen, sondern vor allem auch für Geographen wichtig.

Zum Schluß sei noch auf einen Fortschritt in den Reproduktionsverfahren hingewiesen, nämlich das epochemachende Her-Sol-Verfahren. Es beruht auf der Benützung des sog. Herschel-Effekts und der Solarisation. Durch Verwendung von verschiedenfarbigem Licht und entsprechenden Filtern ist es möglich geworden, das Schwwarz wegzufiltern, vorläufig in einfacheren Fällen. Dadurch können die verschiedenen Farbplatten, ohne Trennung durch Handarbeit, direkt photographisch nach einer fertigen, farbigen Karte erstellt werden. Ein anderes Verfahren, der Ansco Printon Prozeß, erlaubt farbige lithographische Karten farbig zu kopieren.

Die hier besprochene Schrift stellt den Stand der Weltkartographie auf den Zeitpunkt der Abfassung (1949) dar. Darüber hinaus bietet sie, zusammen mit dem zugrunde liegenden Text E/1322/Add. 1 des Conseil économique et social der UNO eine reiche Fülle von Adressen und Literaturangaben. Auf die wertvollen Einzelangaben aus allen Gebieten der Kartographie konnte hier gar nicht eingetreten werden. Die Schrift ist unentbehrlich für jeden, der sich irgendwie mit Kartographie befaßt.

\section{NEKROLOGIE}

\section{HENRI FRANÇOIS PITTIER †}

Am 27. Januar 1950 starb in Caracas, Venezuela, Prof. Dr. Henri François Pittier (*13.8.1857 in Bex). Er war von 1882-1887 Professor der physischen Geographie in Lausanne. 1887 ging er nach Costa Rica, wo er eine äußerst fruchtbare geographische und botanische Tätigkeit entfaltete; die von ihm entworfene Karte von Costa Rica besitzt auch heute noch hohen Wert. Später (19091919) arbeitete er für das amerikanische Landwirtschaftsdepartement in Zentralamerika. Seit 1919 lebte er in Venezuela, wo er als Botaniker eine vielseitige und hochgeachtete Tätigkeit ausübte. H. F. PIrTIER war seit 1899 Ehrenmitglied der Geographisch-Ethnographischen Gesellschaft Zürich.

H. BøsCH

\section{NEUIGKEITEN - NOVA}

Zur Geographie der Vorortsgemeinden. Im Rahmen ortsplanlicher Untersuchungen erschien kürzlich die Arbeit von Peter van BaARsel van Oven, Groningen : Der soziale und wirtschaftliche Aufbau ciner Vorortsgemeinde (Herrliberg/Zürich) (Arbeiten aus dem Institut für Landesplanung ETH Zürich 1951), welche in interessanter Weise das Schicksal städtischer Vororte beleuchtet. Sie ergab folgende Resultate: Die ursprünglich ländlichen Gemeindegebiete werden im Zuge der neuen Entwicklung zu Vorortsgemeinden in verschiedene Siedlungsareale zerlegt. Die einen bewahren mehr bäuerlichen Charakter, die andern werden zu halbstädtischen und städtischen Quartieren. Die Bevölkerungsdichte ist von 1,09 pro ha im Jahre 1836 auf 2,59 pro ha im Jahre 1948 gestiegen (Gemeindedurchschnitt). Im engeren, stark städtisch beeinflußten Gebiet beträgt die Dichte 16,9. Dieses Gebiet umfaßt nur $1 / 8$ des Gemeindeareales, aber $80 \%$ der Gesamtbevölkerung. Die Bevölkerungszunahme beruht hauptsächlich auf Zuzug von freiwilligen Pendlern, die einheimische ländliche Bevölkerung stellt nur $1 / 8$ aller auswärts Arbeitenden der Gemeinde dar, es sind meist unfreiwillige Pendler. Die Zuwanderung von städtischen Familien hat eine Abnahme der Haushaltgrößen von durchschnittlich 5 auf 3 Personen zur Folge. In der ländlichen Bevölkerungsgruppe ist noch ein Männerüberschuß von $14 \%$ festzustellen, während unter der städtischen Bevölkerung 